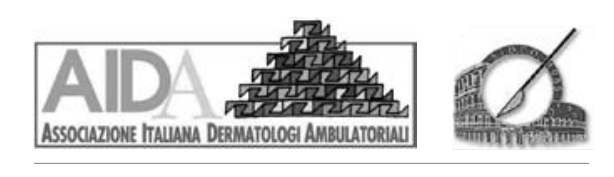

\title{
Peeling chimici: linee guida
}

\author{
Giovanni Labrini ${ }^{1}$, Giuseppe Guerriero ${ }^{2}$, Francesco Luigi Landi ${ }^{2}$, Patrizia Teofoli ${ }^{3}$, Valerio Cirfera ${ }^{4}$
}

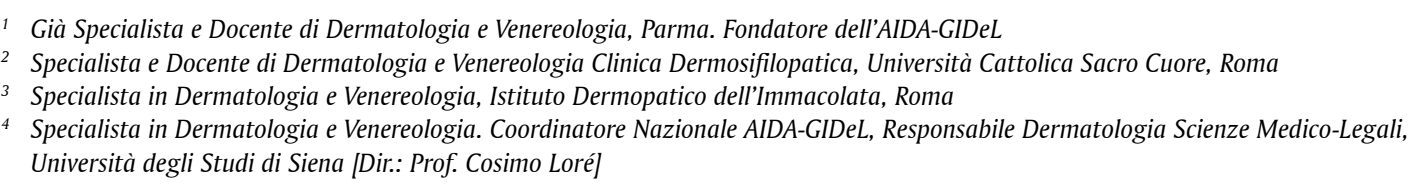

4 Specialista in Dermatologia e Venereologia. Coordinatore Nazionale AIDA-GIDeL, Responsabile Dermatologia Scienze Medico-Legali, Università degli Studi di Siena [Dir:: Prof. Cosimo Loré]

\section{INTRODUZIONE}

La stesura di linee guida del peeling mira a fornire un ausilio professionale, elevando il livello di qualità della prestazione resa, ma nel contempo si prefigge di rendere compartecipi delle finalità e della complessità della procedura i pazienti e $\mathrm{i}$ non addetti ai lavori. La redazione delle linee guida ha impegnato due gruppi di studio, rispettivamente della SIDCO, Società Italiana di Dermatologia Chirurgica e Oncologica e dell'AIDA, Associazione Italiana Dermatologi Ambulatoriali, coordinati dagli Autori. La relazione è stata presentata in occasione dei due Congressi Nazionali, XXI SIDCO, Venezia 26-29 aprile 2006 e XV AIDA, Roma 16-20 maggio 2006 e viene pubblicata sulle riviste delle due società e sui principali siti web di interesse dermatologico.

\section{DEFINIZIONE}

Tra le varie definizioni prospettate, sembra calzante quella proposta dalla Commissione linee guida FISM ("Raccomandazioni per la partecipazione delle Società medico-scientifiche alla produzione, destinazione e valutazione di linee guida di comportamento pratico". QA 1996; 7: 77), secondo la quale le linee guida vanno intese come «raccomandazioni di comportamento clinico, prodotte attraverso un processo sistematico, allo scopo di assistere medici e pazienti nel decidere quali siano le modalità di assistenza più appropriate in specifi- che circostanze cliniche». Con il termine "peeling" (dall'inglese to peel $=$ sbucciare, pelare) si definisce una metodica che consiste nell'applicazione di una o più sostanze chimiche, in immediata o ritardata successione, in grado di indurre la distruzione di aree epidermiche e/o di strati del derma e il successivo processo di rigenerazione tissutale, allo scopo di trattare alcune affezioni cutanee e/o risolverne o migliorarne gli aspetti clinico-estetici. Gli effetti delle sostanze chimiche utilizzate vanno dal semplice distacco dello strato corneo a vistose reazioni infiammatorie del derma; dipendono da svariati fattori e da alcune variabili, in grado di determinare la consistenza dell'effetto di penetrazione, i livelli di profondità raggiungibili e una reazione esfoliativa più o meno marcata. Il peeling viene utilizzato prevalentemente in ambito clinico-estetico e trova essenzialmente indicazione nel trattamento di alcune forme caratterizzate da danno attinico, contribuendo anche ad agire come fattore di prevenzione delle conseguenze del danno indotto da fotoesposizione, comprese le lesioni discheratosiche, potenzialmente precancerosiche.

\section{RAZIONALE}

La definizione di linee guida del peeling si prefigge di contribuire ad identificare e/o precisare:

- le caratteristiche e le proprietà degli agenti chimici;

- le modalità tecniche della loro applicazione;

- le misure precauzionali da attuare;

- le indicazione cliniche e clinico-estetiche; 
- i risultati raggiungibili;

- i trattamenti o le norme da rispettare nelle fasi successive al peeling.

\section{AGENTI CHIMICI INDICATI}

\section{PEELING SUPERFICIALE}

La distruzione tissutale si limita generalmente all'ambito epidermico e non supera comunque le prime propaggini del derma papillare.

Prevede l'utilizzo di:

- resorcina in pasta dal $10 \%$ al $50 \%$;

- soluzione di Jessner o miscela di Combes*, applicabili mediante uno o più passaggi;

- alfa-idrossiacidi, che per contatto prolungato e/o non neutralizzato possono comunque raggiungere livelli più profondi;

- acido salicilico in pasta a varie concentrazioni, per trattare aree diverse dal viso, con possibili effetti avversi (al 50\%, se vengono trattate aree estese, rischio di salicilismo reversibile: tinniti, cefalee, ecc.);

- TCA dal $10 \%$ al $25 \%$. Concentrazioni superiori, specie se applicate in più passaggi, possono penetrare nel derma papillare realizzando un peel medio;

- altri.

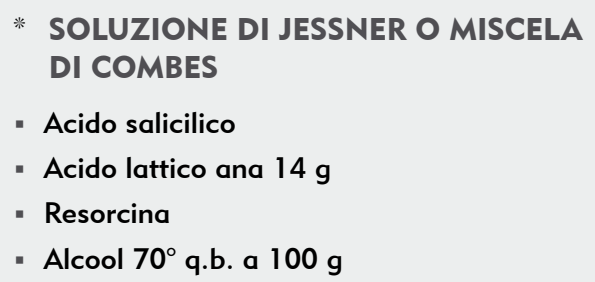

\section{PEELING MEDIO}

La distruzione tissutale prevista è compresa fra il derma papillare e il derma reticolare superiore. È conseguente all'utilizzo di:

- TCA al 50\%, singola applicazione; elevati i rischi di effetti indesiderati e complicanze;

- TCA al 35\%, in applicazioni multiple, fino al conseguimento di frost omogeneo;

- TCA al 35\%, preceduto da $\mathrm{CO}_{2}$ o azoto liquido, o soluzione di Jessner o acido glicolico; effetto sinergico e minori rischi di complican- ze. È possibile aggiungere additivi, come il metil salicilato, per aumentare l'efficacia del TCA al 35\%;

- fenolo $88 \%$ non diluito, che paradossalmente penetra meno profondamente della formula diluita, utilizzata per i peeling profondi;

- altri.

\section{PEELING PROFONDO}

È prevista la distruzione tissutale fino al derma reticolare medio, mediante:

- formula di Baker-Gordon o di Litton ${ }^{* * * *}$, con o senza occlusione;

- altro.

* FORMULA DI BAKER-GORDON

O DI LITTON

- Fenolo al $55 \%$

\section{FASI PREPARATORIE O PRECEDENTI IL PEELING}

- Consenso informato.

- Scelta di detergenti e delipidizzanti adeguati, sulla base della tipologia e della profondità del peeling:

- acetone;

- alcol;

- mix dei due, in ana parti;

- altro.

- Selezione e scelta degli applicatori:

- bastoncini cotonati;

- pennelli;

- garze;

- compresse di garza pressata;

- batuffoli di cotone pressati.

- Appropriata selezione di farmaci topici e sistemici, utilizzabili prima, durante o dopo il peeling:

- antivirali;

- steroidi;

- antibiotici;

- tretinoina;

- antiflogistici;

- analgesici;

- altri. 


\section{PRECAUZIONI PROCEDURALI}

Utilizzare il tipo di peeling e il caustico ideale, valutando il livello teorico di profondità e tutti i fattori valutabili, che verranno descritti successivamente:

- prudente applicazione di qualsiasi agente caustico, usando particolare cautela nelle aree orbitarie, evitando attentamente sgocciolamenti nelle regioni oculari e perioculari;

- tempestiva “diluizione-neutralizzazione”, ove previsto, dei caustici (es.: acido glicoli$\mathrm{co})$;

- redazione di un diagramma esplicativo e illustrativo, da consegnare al paziente, se si utilizzano caustici diversi, su diverse unità estetiche;

- applicazione molto lenta dei peeling a base di fenolo, con intervalli di tempo non inferiori a 15 minuti, tra un'unità estetica e l'altra del viso, per evitare cardiotossicità da rapido assorbimento.

\section{RISCHI CONTROLLABILI}

Evitare:

- utilizzo errato di agente caustico, di concentrazione o di formula;

- utilizzo di prodotti scaduti, divenuti poco $o$, se soggetti a rapida evaporazione, molto concentrati;

- versamento accidentale del caustico addosso al paziente o ai collaboratori di studio;

- altro.

\section{ASPETTI RELATIVI} ALLA SICUREZZA

- Presenza nello studio di sostanze atte, se necessario o laddove previsto, alla diluizione"neutralizzazione" dei caustici:

- acqua (per TCA);

- soluzioni basiche (per alfa/beta-idrossiacidi);

- olio vegetale (per fenolo);

- soluzione per il lavaggio degli occhi.
- Adeguata aerazione e/o ventilazione ambientale.

- Utilizzo di contenitori resistenti agli acidi.

- Rispetto delle norme relative agli studi professionali, ai collaboratori e/o al personale dipendente.

- Altre misure.

\section{CONSIDERAZIONI POST-PEELING}

- Alcuni interventi mirati, se si dovessero rendere necessari, possono agevolare i processi riparativi:

- antibiotici e/o antivirali;

- cortisonici sistemici, topici, intralesionali, veicolati da cerotti e/o in occlusione;

- topici e/o cerotti a base di polimeri siliconici;

- controlli periodici per mantenere i pazienti in osservazione;

- ultrasuonoterapia, prevedendo sedute multiple, allo scopo di "ammorbidire" eventuali esiti cicatriziali;

- altri accorgimenti.

- Alcuni tipi di impacchi, di medicazioni e di topici possono compromettere, accelerare o ritardare i processi riparativi.

- Prescrizioni profilattiche e/o preventive e di supporto e/o terapeutiche, di prodotti contenenti:

- filtri chimici e/o fisici antisolari;

- idrochinone;

- tretinoina;

- alfa-idrossiacidi;

- steroidi;

- antibiotici e/o antivirali;

- associazioni di farmaci;

- altro.

- Accettazione e rispetto da parte dei pazienti della fotoprotezione per tutti i tipi di peeling.

- Controlli post-peeling, con frequenza concordata e adeguata alla tipologia del peeling e/o alle singole necessità cliniche dei pazienti.

- Utilizzabilità (o meno) di tipi particolari di camoufflage o make up.

- Altro. 
In caso di peeling al fenolo, se viene trattata nella stessa seduta più di un'unità cosmetica, devono essere previsti:

- monitoraggio cardiaco

- sedazione im o iv

- analgesia

- infusione lenta di soluzione fisiologica

- piano di gestione delle emergenze

- altre misure

\section{ASPETTI CLINICI} POST-PEELING

Usualmente tipici e previsti gli eventi post-peel, di grado ed intensità variabile, secondo la tipologia del peeling effettuato, possono essere:

- edema;

- eritema;

- vescicolazione;

- presenza di lesioni crostose;

- presenza di lesioni desquamative;

- discromie iper o ipocromiche.

Possibili complicanze post-peeling, tra cui:

- alterazioni pigmentarie e/o lesioni discromiche (tra le quali, alcune possono essere trattate con steroidi, tretinoina, idrochinone, alfa-idrossiacidi e fotoprotettori):

- iperpigmentazioni;

- ipopigmentazioni;

- comparsa o accentuazione delle cosiddette "linee di demarcazione", in corrispondenza delle regioni orbitarie inferiori e/o dei margini mandibolari, mono o bilateralmente;

- accentuazione o sbiadimento del colore di nevi melanocitici;

- presenza associata di alcune o tutte le suindicate evenienze nello stesso paziente;

- eritema prolungato o permanente per mesi (che può richiedere un trattamento steroideo, oltre che fotoprotettivo);

- modificazioni della texture:

- pori dilatati (in genere, transitoriamente);

- accentuazione delle linee di demarcazione (in tutti i tipi di peeling);

- comparsa o peggioramento di teleangectasie, soprattutto in caso di peeling medi o profondi;

- presenza di milia;
- sindrome depressiva post-peel (in cui talvolta è necessario un trattamento specifico);

- altro.

Possibili, anche se meno frequenti, complicanze dei peeling medi e profondi e complicanze rare dei peeling superficiali possono essere:

- infezioni batteriche, virali, fungine;

- tempi ritardati di guarigione;

- ectropion;

- cicatrici di tipo ipertrofico, atrofico o cheloideo, più gravi se insorte in concomitanza 0 a causa di:

- diatesi fibroblastica o comunque predisposizione ereditaria;

- pre-esistente ectropion;

- spessore cutaneo esiguo;

- cute fotodanneggiata o discheratosica;

- recente intervento chirurgico sul viso;

- recente o contemporaneo utilizzo di isotretinoina;

- non rispetto delle norme di fotoprotezione;

- altro.

Possibili complicanze dei peeling profondi al fenolo:

- ipotensione e shock;

- edema laringeo;

- aritmie cardiache;

- altro.

Altre possibili complicanze di tutti i tipi di peeling:

- lesioni atrofiche;

- aumentata sensibilità al freddo dopo criopeel;

- altro.

\section{RIPETIBILITÀ DEL PEELING}

- Peeling superficiali: la ripetibilità è prevista dai protocolli e può essere programmata, a seconda del caustico utilizzato e/o delle evidenze cliniche o clinico-estetiche del paziente, ad intervalli settimanali, bi- o trisettimanali, mensili o secondo valutazione del medico.

- Peeling medi: ripetibilità prevista non prima di 3 mesi.

- Peeling profondi: ripetibilità prevista non prima di 6 mesi possibili variazioni in casi particolari, per specifica valutazione medica. 


\section{ALTRE MODALITÀ O TECNICHE ASSOCIABILI AI PEELING}

- Filler, utilizzabile prima o dopo il peeling. Risulta vantaggioso un intervallo da 2-3 settimane a 2-3 mesi o più tra un peeling e l'altro, a seconda del tipo di peel, per favorire l'integrazione del filler nella cute.

- Utilizzo topico pre- o post-peel di tretinoina e/o idrochinone.

- Dermoabrasione, laserabrasione.

È prudente un intervallo di almeno 3-6 mesi prima o dopo il peel per:

- crioterapia;

- altro.

\section{CRITERI CLINICO-DIAGNOSTICI}

\section{CLINICI \\ 1. Anamnesi \\ - generale \\ - mirata \\ 2. Esame obiettivo}

\section{ESAMI DIAGNOSTICI}

\section{CRITERI CLINICI}

\section{Anamnesi}

- Generale.

- Mirata per valutare eventuali controindicazioni non assolute.

\section{Controindicazioni non assolute}

Relative a tutti i peeling:
- herpes simplex in fase attiva, in area o aree da trattare;

- pregressi interventi chirurgici interessanti il viso o aree facciali, caratterizzati da tecniche di scollamento (lifting, blefaroplastica, microliposuzione, ecc);

- pregressa radioterapia, limitante la riepitelizzazione a partenza annessiale;

- recente trattamento sistemico con isotretinoina;

- diatesi fibroblastica;

- terapie in atto, controindicate per supposti rallentamenti e/o ritardi della fase riparativa;

- malattie autoimmunitarie, per rischio elevato di:

- infezioni;

- rallentamento dei processi riparativi;

- ipercromie post-peeling;

- altro.

Relative ai peeling medi e profondi:

- fototipi alti (IV-VI secondo Fitzpatrick), per la possibilità di discromie ipercromiche;

- altro.

Fenolo:

- anamnesi positiva per cardio e/o nefro-epatopatie, se è previsto il trattamento di più di 1 unità estetica;

- altro.

È sempre necessario valutare comunque che $i$ candidati al peeling siano animati da aspettative realistiche e manifestino adeguata disponibilità psicofisica a tollerare il trattamento e la convalescenza. In pazienti ansiosi, può essere prudente praticare uno spot test per valutare (ma non è un rilievo da ritenere sicuramente predittivo e probante) un'eventuale esito pigmentario.

È anche indispensabile verificare la disponibilità e la capacità del paziente a effettuare eventuali medicazioni o trattamenti specifici nel post-peel, come ad esempio il camoufflage, anche per tempi lunghi, in caso di discromie persistenti

\begin{tabular}{|cccc|}
\hline \multirow{2}{*}{ Fototipo } & Sensibilità agli UV & \multicolumn{2}{c|}{ Reazione all'esposizione solare } \\
\cline { 3 - 4 } & Elevata & Scottatura & Abbronzatura \\
\hline I & Elevata & Sempre, con facilità & Poco \\
\hline II & Media & Sempre, moderata & Gradualmente \\
\hline III & Scarsa & Minima & Sempre, con rapidità \\
\hline IV & Minima & Raramente & Sempre, con rapidità \\
\hline V & Nulla & Mai & Sempre, con rapidità \\
\hline VI & &
\end{tabular}

Tabella I. Classificazione di Fitzpatrick 


\begin{tabular}{|c|c|}
\hline Disturbi pigmentari & $\begin{array}{l}\text { - Melasma } \\
\text { - Esiti pigmentari post-infiammatori } \\
\text { - Altro }\end{array}$ \\
\hline Fotoinvecchiamento & $\begin{array}{l}\text { - Cheratosi attiniche } \\
\text { - Lentigo solaris } \\
\text { - Discromie pigmentarie } \\
\text { - Elastosi solare } \\
\text { - Dermatoeliosi } \\
\text { - Rughe elastotiche } \\
\text { - Altro }\end{array}$ \\
\hline Rughe & $\begin{array}{l}\text { - Attiniche } \\
\text { - Dinamiche } \\
\text { - Da sonno: sleeping creases }\end{array}$ \\
\hline Altri quadri & $\begin{array}{l}\text { - Cicatrici superficiali } \\
\text { - Cheratosi post-radiazioni } \\
\text { - Acne volgare e rosacea, forma cicatriziale } \\
\text { e non cicatriziale } \\
\text { - Verruche volgari } \\
\text { - Milia } \\
\text { - Iperplasia sebacea } \\
\text { - Altro }\end{array}$ \\
\hline
\end{tabular}

Tabella II. Patologie cutanee trattabili con il peeling

\section{Esame clinico}

Occorre ovviamente prendere in considerazione:

- l'aspetto fisico generale;

- il fototipo (I-IV) secondo la classificazione di Fitzpatrick (Tabella I)

- il grado di fotoinvecchiamento, per scegliere la tipologia di peeling e il caustico da utilizzare, secondo il livello di profondità che si intende raggiungere;

- la densità e lo stato di attività delle ghiandole sebacee;

- il grado di lassità cutanea delle regioni orbitarie;

- la presenza di cicatrici ipertrofiche e/o cheloidee su tutto l'ambito cutaneo, oltre che sul viso;

- la presenza di infezioni nelle aree da sottoporre a peeling;

- altre dermopatie;

- una valutazione fotografica delle aree da trattare, per poter poi effettuare una comparazione dell'obiettività pre- e post-peeling;

- la corretta indicazione clinica o clinico-estetica.

Possono essere trattate con peeling chimico alcune forme cutanee, riportate nella Tabella II, inquadrate in gruppi di riferimento.

\section{Criteri diagnostici}

- Eventuale biopsia, se vi è indicazione.

- ECG, esami ematochimici, in funzione di un peeling con fenolo.

- Altro.

\section{Requisiti dell'operatore}

Obbligatori:

- laurea in medicina e chirurgia e abilitazione professionale.

Auspicati:

- specializzazione in dermatologia o in chirurgia plastica;

- frequenza ad almeno tre corsi e preferibilmente training certificato presso riconosciuti esperti del settore.

Il medico deve comunque avere piene conoscenze dell'anatomia, dell'istologia e della fisiopatologia della cute e del tessuto sottocutaneo e, più in specifico, dei seguenti temi:

- anatomia cutanea delle unità cosmetiche del viso;

- differenze strutturali e funzionali delle varie aree corporee;

- fisiopatologia del danno fotoindotto;

- varie fasi della riparazione tissutale, successive a un peeling:

- processi coagulativi e infiammatori;

- angiogenesi;

- formazione di tessuto di granulazione;

- riepitelizzazione;

- rimodellamento del collagene.

È fondamentale che il medico abbia esperienza clinico-pratica, maturata durante la specializzazione in dermatologia o in chirurgia plastica, e/o abbia frequentato corsi specialistici teorico-pratici per acquisire la conoscenza di tecniche peeling e di procedure comprendenti:

- applicazione della varietà dei caustici;

- applicazioni multiple di caustici;

- metodi e tecniche occlusive (in caso di utilizzo del fenolo);

- esperienza dermochirurgica di base.

\section{AVVERTENZE}

- La stesura delle linee guida si basa sullo stato delle attuali conoscenze e riflette dati desunti dalla letteratura scientifica di riferimento. In ogni caso i dati vanno sempre analizzati e interpretati attentamente, con adeguato senso critico. Studi successivi potranno indurre 
modifiche o cambiamenti delle conclusioni o raccomandazioni riportate in questo documento.

- Il rispetto delle linee guida non assicura in ogni caso la certezza di un trattamento sicuramente soddisfacente ed esente da rischi.

- La valutazione definitiva, relativa alle caratteristiche e alle proprietà di ogni specifica procedura peeling, compete in ogni caso al medico, sulla base della valutazione clinica e delle indicazioni relative al singolo paziente, nella definita circostanza temporale, coinci- dente con il momento dell'effettuazione del peeling.

- L'aggiunta di sostanze, con l'intento di potenziare il caustico di base, non consente allo stato attuale valutazioni relative a possibili benefici e/o rischi.

- Lutilizzo di peeling commerciali impegna sul piano etico, culturale e medico-legale gli utilizzatori e i produttori.

- Qualcuna delle sostanze indicate nel pre- e post-peel potrebbe allo stato attuale non essere dispensabile in farmacia, se non in forma galenica magistrale.

\section{BIBLIOGRAFIA}

1. Drake LA, Dinehart SM, Goltz RW, Graham GF, Hordinsky MK, Lewis C et al. Guidelines of care for chemical peeling. Guidelines/ Outcomes Committee: American Academy of Dermatology. J Am Acad Dermatol 1995; 33: 497-503

2. Drake LA, Ceilley RI, Cornelison RL, Dinehart SM, Dorner W, Goltz RW et al. Guidelines of care for office surgical facilities. Part I. J Am Acad Dermatol 1992; 26: 763-5

3. Drake LA, Ceilley RI, Cornelison RL, Dinehart SM, Dorner W, Goltz RW et al. Guidelines of care for office surgical facilities. Part II. Self-Assessment checklist. J Am Acad Dermatol 1995; 33: 265-70

4. Griffiths CE. The british association of dermatologists guidelines for the management of skin disease. Br J Dermatol 1999; 141: 396-7

5. Stegman SJ, Tromovitch TA, Glogau RG. Chemical peels. In: Cosmetic dermatologic surgery. Chicago: Year Book Medical Publishers, 1990; pp. 35-58

6. Baker TJ, Gordon HL. Chemical peel with phenol. In: Epstein E, Epstein E Jr. Skin surgery. Philadelphia: WB Saunders, 1987; pp. $423-38$

7. Brody HJ. Chemical peeling. St Louis: Mosby-Year Book, 1992

8. Brody HJ. Special issue: chemical peels. J Dermatol Surg Oncol 1989; 15: 916-1024

9. Brody HJ, Alt TH. Chemical peeling. In: Coleman WP, Hanke CW, Alt TH. Cosmetic surgery of the skin. Philadelphia: BC Decker, 1991; pp. 65-88

10. Labrini G. Peeling chimici. Dermatologia Ambulatoriale 2005; 1-2: 24-32

11. Brody HJ, Hailey CW. Medium-depth chemical peeling of the skin: a variation of superficial chemosurgery.J Dermatol Surg Oncol 1986; 12: 1268-75

12. Matarasso SL, Glogau RG. Chemical face peels. In: Thiers BH, McCollough E., Langston PR. Dermabrasion and chemical peel: a guide for facial plastic surgery. New York: Thieme Medical Publishers, 1988; pp. 53-112

13. O’Donoghue MN. Dermatologic Clinics. Philadelphia: WB Saunders, 1991; pp. 131-50

14. Roenigk R.K. Retinoids, dermabrasion, chemical peel and keloids. In: Reonigk RK, Roenigk HH Jr. Surgical dermatology: advances in current practice. St. Louis: Mosby, 1993; pp. 376-83

15. Labrini G. Chemical face peels: management of side effects and complications. EADV 3d Spring Symposium, Sofia: 19-22 May 2005. Book of Abstract, pp. 168-169

16. Ayres S $3^{\text {rd }}$. Superficial chemosurgery in treating aging skin. Arch Dermatol 1962; 85: 125-33

17. Clark RE, Weingold OH, Hirsh E. et al. TCA chemical peel effective for extensive actinic keratoses. Skin Allergy News 1991; 22: 34

18. Collins PS. The chemical peel. Clin Dermatol 1987; 5: 57-74

19. Labrini G. Peeling chimico: proposta di una scheda paziente anamnestico-procedurale. Dermatologia Ambulatoriale 2004; 4: 50-4

20. Labrini G. Side effects and complications of chemical peeling. Jeadv 2003; 17: 465

21. Kligman AM, Baker TJ, Gordon HL. Long-term histologic follow-up of phenol face peels. Plast Reconstr Surg 1985; 75: 652-9

22. Van Scott EJ, Yu RJ. Alpha hydroxy acids: procedures for use in clinical practice. Cutis 1989; 43: 222-8

23. Guerriero G, Pagliarello C, Landi FL. Acido mandelico: recenti acquisizioni in tema di "peeling" chimico. Il Dermochirurgo 2005; $1: 21-3$

24. Landi FL, Guerriero G, Pagliarello C, Paradisi A, Pizarro N. Validità di un trattamento "peeling" con acido piruvico al $40 \%$ in pazienti acneici con diatesi atopica. Esperienze Dermatologiche 2005; 4: 257-60 
25. Guerriero G, Ciasulli A, Pagliarello C, Landi FL. Studio istopatologico e valutazione dell'attività biochimica dell'acido piruvico (PA) vs acido tricloroacetico (TCA) nei "peelings" chimici con finalità dermoestetiche. Il Dermochirurgo 2005; 1: 10-6

26. Stegman SJ. A comparative histologic study of the effects of three peeling agents and dermabrasion on normal and sun-damaged skin. Aesthet Plast Surg 1982; 6: 123-35

27. Brown AM, Kaplan LM, Brown ME. Phenol-induced histological skin changes: hazards, technique, and uses. BrJ Plast Surg 1960; 13: $158-69$ 